\title{
Curing behaviors, mechanical properties, dynamic mechanical analysis and morphologies of natural rubber vulcanizates containing reclaimed rubber
}

https://doi.org/10.1515/epoly-2019-0051

Received March 11, 2019; accepted June 27, 2019.

\begin{abstract}
Ground tire rubber (GTR) with crosslinked structure shows hardly any plasticity and processability, giving rise to its relatively poor properties. The thermalmechanical shearing devulcanization can effectively break the three-dimension crosslinked network structure and restore a certain extent of plasticity and processability. In this work, reclaimed rubber (RR) was obtained by thermalmechanical shearing devulcanization as a potential filler for natural rubber. The vulcanization process, mechanical properties, microstructure of $\mathrm{RR} /$ natural rubber (NR) compounds composites were investigated. It was found that the NR vulcanizates containing $30 \mathrm{phr}$ of $\mathrm{RR}$ as filler showed the higher tensile strength and elongation at break. Microstructural characterization analysis on a sample containing $30 \mathrm{phr}$ of $\mathrm{RR}$ revealed the existence of strong interactions between the natural rubber matrix and RR, suggesting that RR and NR appeared to be wellmatched, undergoing simultaneous curing and forming a continuous phase.
\end{abstract}

Keywords: ground tire rubber (GTR); reclaimed rubber; natural rubber; shearing devulcanization; mechanical properties

\footnotetext{
* Corresponding authors: Xipo Zhao and Shaoxian Peng, Hubei Provincial Key Laboratory of Green Materials for Light Industry, Collaborative Innovation Center of Green Light-weight Materials and Processing, School of Materials and Chemical Engineering, Hubei University of Technology, Wuhan, Hubei, 430068, China, e-mail:xpzha0123@163.com, psxbb@126.com Huan Hu, Dengke Zhang, Zhen Zhang and Yiming Sun, Hubei Provincial Key Laboratory of Green Materials for Light Industry, Collaborative Innovation Center of Green Light-weight Materials and Processing, School of Materials and Chemical Engineering, Hubei University of Technology, Wuhan, Hubei, 430068, China
}

\section{Introduction}

With the fast development of the auto industry, the production of waste rubbers is increasing rapidly (1). In 2012, Sienkiewicz et al. (2), quoted the annual global production of tires is 1.4 billion units, which corresponds to an estimated 17 million tons of used tires each year. Many ways have been adopted to recycle and reuse waste tires to solve the problem $(3,4)$.

The reclaimed rubber (RR) and ground tire rubber (GTR) have been commonly applied in the rubber industry as rubber substitutes and fillers for rubber composites and/or blends, giving rise to better applications prospects for RR and GTR. Unlike in the GTR, RR contains carbon black and small molecules, and the small molecules could be used as a plasticizer, resulting in the improved dispersion properties of the filler. In addition, RR with special structures could be used as an elastic filler for the NR, showing better compatibility than the inorganic fillers. NR vulcanizates containing RR and GTR have been investigated (5), and it was found that compared to GTR/NR compounds, RR/NR compounds had much better tensile properties, which are comparable to or even better than those of the original vulcanized rubbers, illustrating the significant benefit of thermal-mechanical shearing devulcanization. A recent investigation of a GTR/carbon black mixture as a hybrid filler for NR (6), showed that through the partial replacement of the carbon black active filler, with GTR has a favorable influence on the mechanical properties and processability of the vulcanizates.

Various regeneration processes strongly impact the performance of reclaimed rubber by destroying the key S-S and C-S bonds and easily damaging the rubber molecules chains to a large extent. In preliminary works $(7,8)$, we had obtained RR with a high gel content and low crosslinking density by thermal-mechanical shearing desulfurization. The macromolecular chain of the reclaimed rubber was kept relatively intact, and the performance of the secondary cured rubber was improved. The blends incorporating RR 
could effectively enhance the characteristics of RR/NR compounds and accelerated the curing rate. These blends also help to form a new crosslinked network structure and obtain high performance RR/NR compounds.

Natural rubber (NR) has been used in numerous industrial rubber products, due to its excellent tensile strength and good abrasion resistance, NR is suitable for production of o-rings, seals, tires, convey or belts, etc. (9). Although NR was known to exhibit many outstanding properties, in most cases, it is necessary to add reinforcing fillers into NR in order to obtain the properties required for the specific application. In fact, a wide variety of particulate fillers have been used in the rubber industry for various purposes (10). The NR composites with good mechanical and functional properties can be prepared by waste free technology and reasonable economy of material manufacturing. In this research, the RR obtained from thermal-mechanical shearing devulcanization has been blended with NR. The vulcanization process, mechanical properties and microstructure of RR/NR were investigated.

\section{Experimental}

\subsection{Materials and compounding ingredients}

$\mathrm{NR}$, grade SVR3L, with mooney viscosity $\left(\mathrm{ML}[1+4] 100^{\circ} \mathrm{C}\right.$ of 72 , relative density of $0.93 \mathrm{~g} / \mathrm{cm}^{3}$, ash content of $0.42 \%$ and polyisoprene content of $91-94 \%$ was supplied by the Creative Polymers Co., Ltd. (Bangkok, Thailand). GTR powder, with the particle size of 30 mesh, volatile component content of $6 \mathrm{wt} \%$, rubber content of $58 \mathrm{wt} \%$, carbon black content of $30 \mathrm{wt} \%$, and ash content of $6 \mathrm{wt} \%$ was purchased from the Nantong Huili Rubber Co. (China). Other curing ingredients and additives such as triethylene amine, dioctyl phthalate, petroleum resin, zinc oxide (ZnO), stearic acid (SA), N-Cyclohexyl-2-benzothiazole sulfonamide (CZ), tetramethyl thiuram disulfide (TMTD), N-1,3-dimethylbutyl-N'-phenyl-p-phenylenediamine (6PPD) and sulfur were purchased from Bayer (M) Sdn. Bhd. Using $100 \mathrm{phr}$ of rubber as the reference, the amount of sulfurizing agent and reagents are as follow: $100 \mathrm{phr}$ rubber, 5.0 phr ZnO, 2.0 phr SA, 0.5 phr CZ, 0.2 phr TMTD, and $2.0 \mathrm{phr}$ sulfur.

\subsection{Sample preparation}

Reclaimed rubber was prepared as follows: $100 \mathrm{phr}$ ground tire rubber, $4 \mathrm{phr}$ triethylene amine as reclaiming agent, $2 \mathrm{phr}$ dioctyl phthalate as reclaiming reagent, $2 \mathrm{phr}$ petroleum resin as activator and 5 phr deionized water, mixed with a high speed mixer. The mixer of ground tire rubber, reclaiming agent and all reagents were activated and swelled at $220^{\circ} \mathrm{C}$ for $2.5 \mathrm{~h}$ in high pressure reactor. And then used a torque rheometer mixer at $180^{\circ} \mathrm{C}$ for $20 \mathrm{~min}$, and finally obtain the reclaimed rubber with gel fraction of $68.6 \%$ and crosslink density of $0.33 \mathrm{~mol} / \mathrm{dm}^{3}$.

All RR/NR or GTR/NR compounds were prepared using the compositions given in Table 1. For comparison raw NR compounds were also prepared. First, in the roll gap of $1 \mathrm{~mm}$, the temperature of $50^{\circ} \mathrm{C}$ on the two-roll mixing machine mixing NR and RR, kneading evenly after 5 times triangular bag, followed by the formula required by adding $\mathrm{ZnO}$, SA, accelerator CZ and TMTD, left and right cutting knife 2 times, to be eaten completely after adding the curing system, mixing even after adjusting the roller from the film stand. And then the rubber compound in the flat vulcanizing machine molded under $150^{\circ} \mathrm{C}$ and $10 \mathrm{MPa}$ to obtain $2 \mathrm{~mm}$ thick sheet.

\subsection{Characterizations}

\subsubsection{Cure characterizations}

The vulcanization process was analyzed according to PN-ISO 3417 at $150^{\circ} \mathrm{C}$, using a rheometer without an oscillating rotor. The oscillation angle was $3^{\circ}$ and the torque range was $0-100 \mathrm{dNm}$. The cure rate values were calculated according to:

$$
V_{c}=\frac{1}{t_{c 90}-t_{s 2}}
$$

where $t_{c 90}$ is the optimum vulcanization time $(\min )$ and $t_{\mathrm{s} 2}$ is the scorch time (min).

\subsubsection{Static mechanical properties}

Static mechanical properties (tensile strength, elongation at break and shore hardness) of the RR(GTR)/NR vulcanizates were determined in accordance with ISO 37 using dumbbell-shaped samples. Tensile tests

Table 1: The ratios of NR and RR or GTR.

\begin{tabular}{lccccccc}
\hline Samples & 1 & 2 & 3 & 4 & 5 & 6 & 7 \\
\hline NR/phr & 100 & 90 & 70 & 50 & 30 & 10 & 0 \\
RR or GTR/phr & 0 & 10 & 30 & 50 & 70 & 90 & 100 \\
\hline
\end{tabular}


were performed using CMT-4204 electric tensile tester (Shenzhen SANS, China) at a speed of $10 \mathrm{~mm} / \mathrm{min}$. Shore hardness type A was determined using a durometer (Zwick 3130; Germany) in accordance to ISO 7619.

\subsubsection{Dynamic mechanical properties}

The dynamic mechanical properties of the RR/NR or GTR/NR blends were determined using a dynamic mechanical analyzer (DMA) (Q800; TA; USA) in a dualcantilever deformation mode. It was used to study the relationship between modulus and temperature of the RR/NR or GTR/NR compounds. The test samples were rectangular with the length, width and thickness of 40,10 and $2 \mathrm{~mm}$, respectively. All tests were performed from $-80^{\circ} \mathrm{C}$ to $20^{\circ} \mathrm{C}$ at a heating rate of $4^{\circ} \mathrm{C} / \mathrm{min}$ and the frequency of $1 \mathrm{~Hz}$.

\subsubsection{Morphological analysis}

The morphologies of the RR/NR and GTR/NR compounds were examined by scanning electron microscope (SEM) (S-6390; Hitachi, Tokyo, Japan) at an accelerating voltage of $30 \mathrm{kV}$. The hot-pressed samples were cryo-fractured in liquid nitrogen for $4 \mathrm{~h}$. Then, the cryo-fractured samples were coated with Pt-Pd.

\section{Results and discussion}

\subsection{Cure characteristics of RR/NR compounds}

The effects of different proportions on the vulcanization process parameters of RR and NR are described by the data presented in Table 2 . The torque increased continuously with increasing the amount of $\mathrm{RR}$, and the cure rate also increased, whereas the scorch time and optimum cure time decreased continuously. This could be explained as due to the fact that reclaimed rubber was obtained by the desulfurization and regeneration of the ground tire rubber powder, and contains carbon black and small molecules, Additionally, the specially structured reclaimed rubber can be used as an elastic filler in NR, because reclaimed rubber and natural rubber showed better compatibility with natural rubber than the other fillers. With the increase in the RR content, the carbon black content increased as well, leading to the increase in the values of the minimum and maximum torques of the RR/NR compounds. The presence of carbon black also accelerated curing reaction rates. While the free sulfur radicals accelerated the concerted reaction with the curing system, resulting in the enhancement of the cure rate.

\subsection{Mechanical properties of RR (GTR)/NR compounds}

Figures 1-4 show the tensile properties, $100 \%$ modulus and hardness, respectively, of the vulcanizates of the RR/NR and GTR/NR blends plotted versus the RR and GTR concentration. The tensile strength and elongation at break decreased continuously with increasing $R R$ and GTR content. This could be explained as due to the fact that GTR was used as an inert filler in the GTR/NR compounds, and therefore an increase in the GTR content, made the material more prone to reunite, resulting in a significant reduction in the tensile strength of the GTR/NR compounds. Unlike the ground tire rubber powders, reclaimed rubber contained carbon black and small molecules, that could reinforced the rubber compounds; however, with the increasing amount of reclaimed rubber, the amount of broken rubber networks and filler particles of reclaimed rubber also increased;

Table 2: Cure parameters for RR/NR compounds.

\begin{tabular}{lccccccc}
\hline Cure parameters & \multicolumn{7}{c}{ RR/NR } \\
\cline { 2 - 7 } & $\mathbf{0 / 1 0 0}$ & $\mathbf{1 0 / 9 0}$ & $\mathbf{3 0 / 7 0}$ & $\mathbf{5 0 / 5 0}$ & $\mathbf{7 0 / 3 0}$ & $\mathbf{9 0 / 1 0}$ & $\mathbf{1 0 0 / 0}$ \\
\hline $\mathrm{M}_{\mathrm{L}} / \mathrm{dN} \cdot \mathrm{m}$ & 0.38 & 0.88 & 1.01 & 1.04 & 1.07 & 2.48 & 2.51 \\
$\mathrm{M}_{\mathrm{H}} / \mathrm{dN} \cdot \mathrm{m}$ & 8.69 & 11.33 & 9.87 & 12.08 & 12.11 & 18.17 & 18.03 \\
$\mathrm{M}_{\mathrm{H}}-\mathrm{M}_{\mathrm{L}} / \mathrm{dN} \cdot \mathrm{m}$ & 8.31 & 10.45 & 8.86 & 11.07 & 11.03 & 15.69 & 15.52 \\
$\mathrm{t}_{\mathrm{c} 10} / \mathrm{min}$ & 7.39 & 6.72 & 6.23 & 5.91 & 4.52 & 3.91 & 3.14 \\
$\mathrm{t}_{\mathrm{c} 90} / \mathrm{min}$ & 21.32 & 18.67 & 16.34 & 15.6 & 13.21 & 10.67 & 9.13 \\
$\mathrm{~V}_{c} / \mathrm{min}^{-1}$ & 0.0718 & 0.0837 & 0.0989 & 0.1031 & 0.1150 & 0.1479 & 0.1666 \\
\hline
\end{tabular}




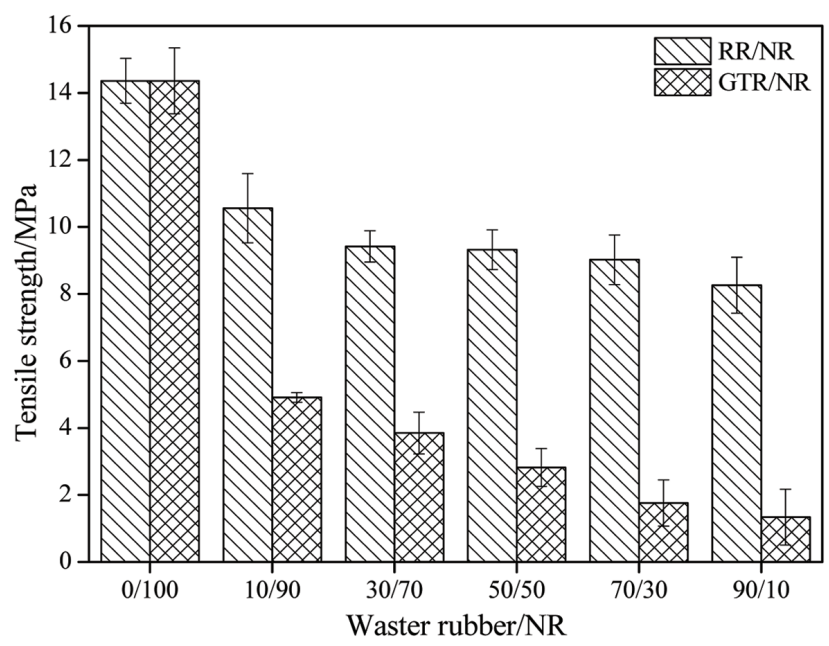

Figure 1: Tensile strength of RR(GTR)/NR compounds containing different contents.

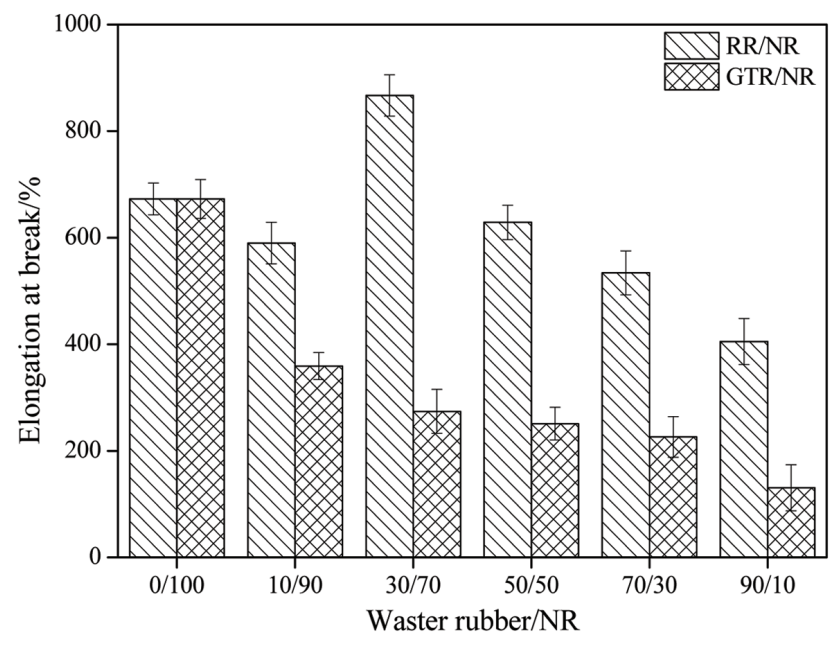

Figure 2: Elongation-at-break for different contents of RR(GTR)/NR.

so that the rubber compounds were easily damaged due to the destructive effect of stress concentration points. Additionally, the hydrocarbon content of the reclaimed rubber increased with increasing reclaimed rubber content, so that the two phases could not form a uniform dispersion, and the interfaces between the rubber compounds acted as new failure points; Therefor, tensile strength decreases continuously with increasing RR content. On the other hand, 100\% modulus and hardness increased continuously with increasing RR and GTR content. This could be explained as due to the significant breaking of the reclaimed rubber macromolecule chains, so that even though the reclaimed rubber content increased, the rubber compounds filler content increased and the rigidity of the rubber compounds filler particles increased the hardness and 100\% modulus, However, a

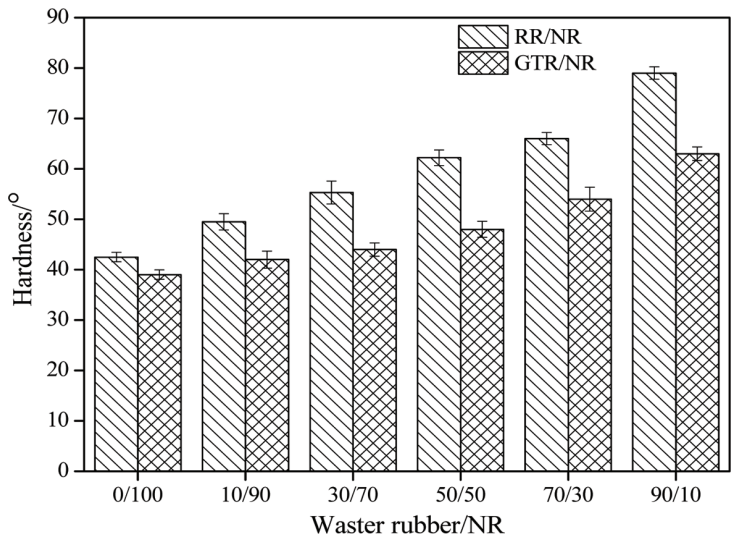

Figure 3: Hardness for different contents of RR(GTR)/NR compounds.

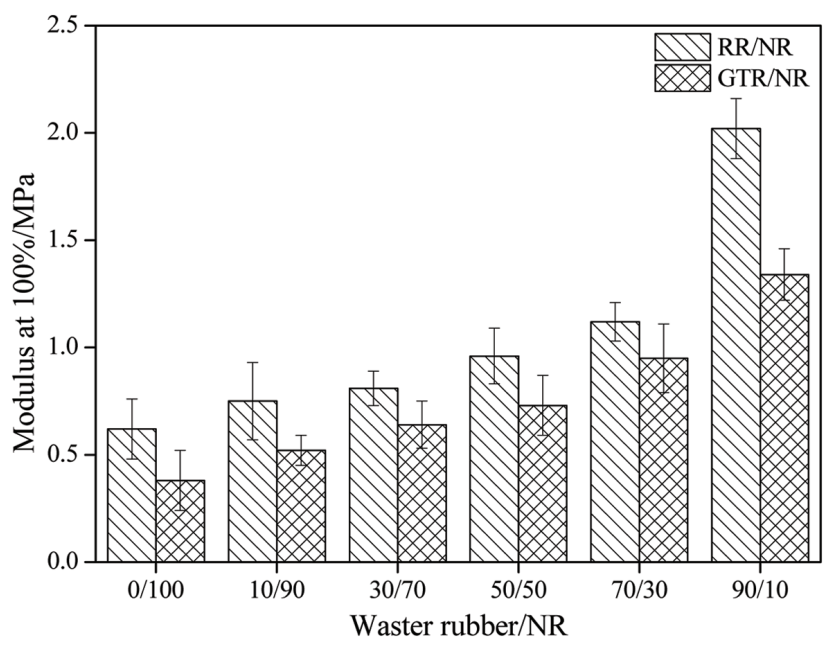

Figure 4: $100 \%$ moduli for different contents of RR(GTR)/NR compounds.

cross-linking network of could not be formed completely, resulting in the decrease of the elongation at break.

\subsection{Dynamic properties of $R R / N R$ compounds}

DMA has proved to be useful for the investigation of viscoelastic behaviors of rubber compounds (11). Here we focus on the temperature variations of the viscoelastic properties of RR/NR compounds that are always expressed by the storage modulus, loss modulus and the $\tan \delta$ parameter.

Figure $5 \mathrm{a}$ shows the storage modulus-temperature curves for different proportions of RR/NR vulcanizates. The storage modulus is the maximum energy that can be stored in a period of time and can provide a reference value for the rigidity of the rubber, thus reflecting the 

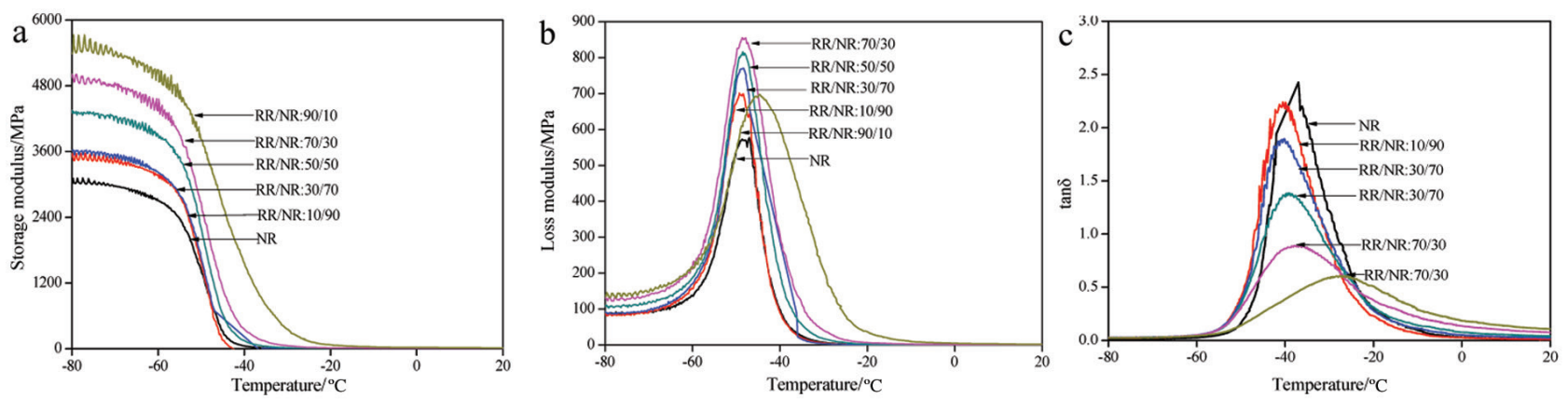

Figure 5: Dynamic mechanical thermal properties of RR/NR vulcanizates.

elastic properties of the polymeric materials $(12,13)$. As the temperature increases, the molecular chains begin to thaw, In the rubbery area, the molecular segments are free to move and the friction to be overcome is small, and the friction energy is reduced, The internal energy consumption is very small, resulting in no significant changes in storage modulus (14). Figure 5a shows that the RR/NR compounds filled with $90 \mathrm{phr}$ of RR loading showed higher storage modulus values than compounds with other compositions. This is mainly due to the thermal-mechanical shear desulfurization of RR with linear plasticity and machinability, and the structure of $\mathrm{RR}$ and NR is more similar, RR can be evenly dispersed in NR matrix, and RR can re-vulcanization molding, thus, resulting in the storage modulus significantly increased of $\mathrm{RR}$ and NR compounds. Additionally, RR/NR compounds filled with small amount of RR show higher storage modulus values than the raw NR vulcanizates, resulting in the stronger RR-NR interactions (15).

Figure $5 \mathrm{~b}$ shows the loss modulus-temperature curves for different proportions of RR/NR compounds, the loss modulus refers to the energy lost by the material in the event of a viscous deformation that reaction the viscous nature of polymer material (16). With the increase of the amount of RR, the loss modulus of RR/NR compounds changed significantly. The loss modulus values of the $\mathrm{RR} / \mathrm{NR}$ compounds filled with $70 \mathrm{phr}$ of RR content increased significantly than those for other compositions due to the hindrance of the molecular motion.

Figure $5 \mathrm{c}$ shows the $\tan \delta$-temperature curves for different proportions of RR/NR compounds. Tan $\delta$ is the ratio of the loss modulus to the storage modulus, and is an indicator by the dynamic behavior of RR/NR compounds (11). In general, the main causes of compound damping are: (a) the properties of the matrix and the filler; (b) the nature of the interface; (c) the friction damping or delamination resulting from the sliding of the unbounded area between the filler and the substrate; and (d) energy dissipation in the matrix crack region. The damping peaks always occur in the glass transition region and are related to the movement of pendant groups or low molecular weight units and molecular chains in the rubber. Thus, the higher damping peak corresponds to a higher degree of molecular mobility (17). With the increase of the content of RR, RR/NR compounds damping performance decreased significantly, thus, giving rise to a lower and broader damping peak. This is due to RR acting as a barrier to the mobility of the rubber molecular chains, restricting the rubber segments motion through the strong interaction between the RR and NR molecular chains, and indicating the reinforcing nature of the filler with NR (18). The temperature of the $\tan \delta$ peak is the glass transition temperature $(\mathrm{Tg})$, that can be used to indicate the degree of the reinforcement of the rubber (19). In this work, the amount of RR has no significant effect on $\mathrm{Tg}$ value of RR/NR compounds, The $T g$ value of the RR/NR blends filled with $90 \mathrm{phr}$ of RR content became closer to the high temperature zone than the those for the other compositionss. This could be explained as follows. As the temperature increases, some RR and NR crosslinked with each other, a certain degree of infiltration reactions occurred, and the macromolecular chains became closely intertwined, thus enhancing the heat resistant properties of RR/NR composites (20).

\subsection{Morphological studies of RR/NR compounds}

Figures 6 and 7 show the microstructure for different proportions of GTR/NR and RR/NR compounds. Examination of the images of the fracture surfaces of the GTR/NR compounds presented in Figures 6a-c at high magnification, revealed that the GTR powder have no plasticity and secondary processing performance, and the compatibility with the NR is poor, easy to agglomerate powder together to form a defect result, therefore 

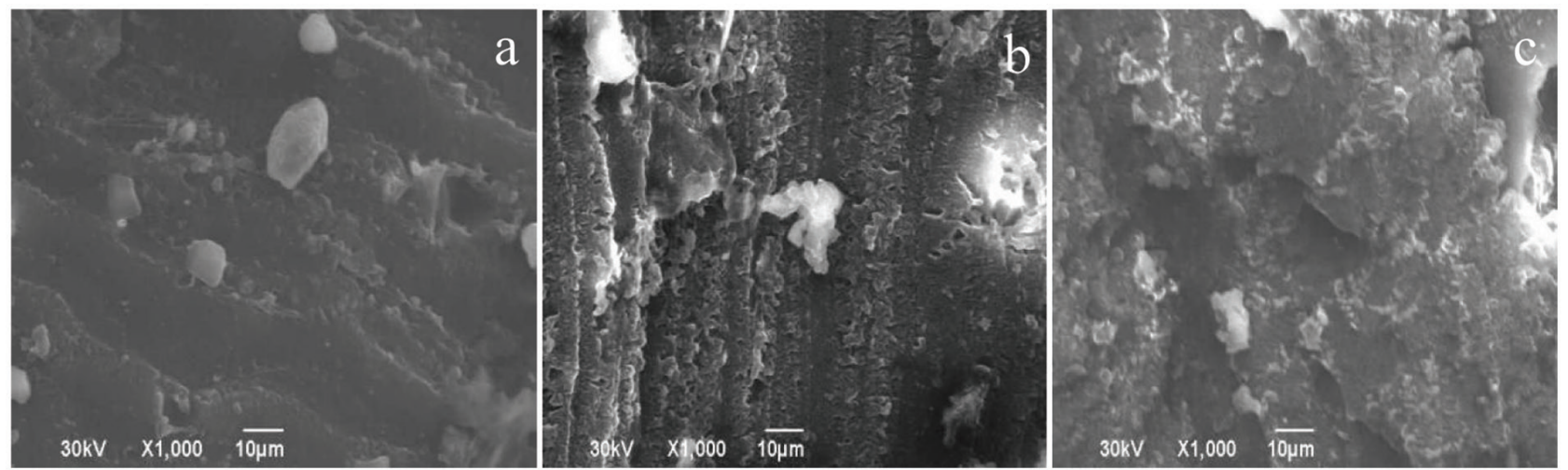

Figure 6: SEM images of GTR/NR: (a) 30/70, (b) 50/50, (c) 70/30 blends.
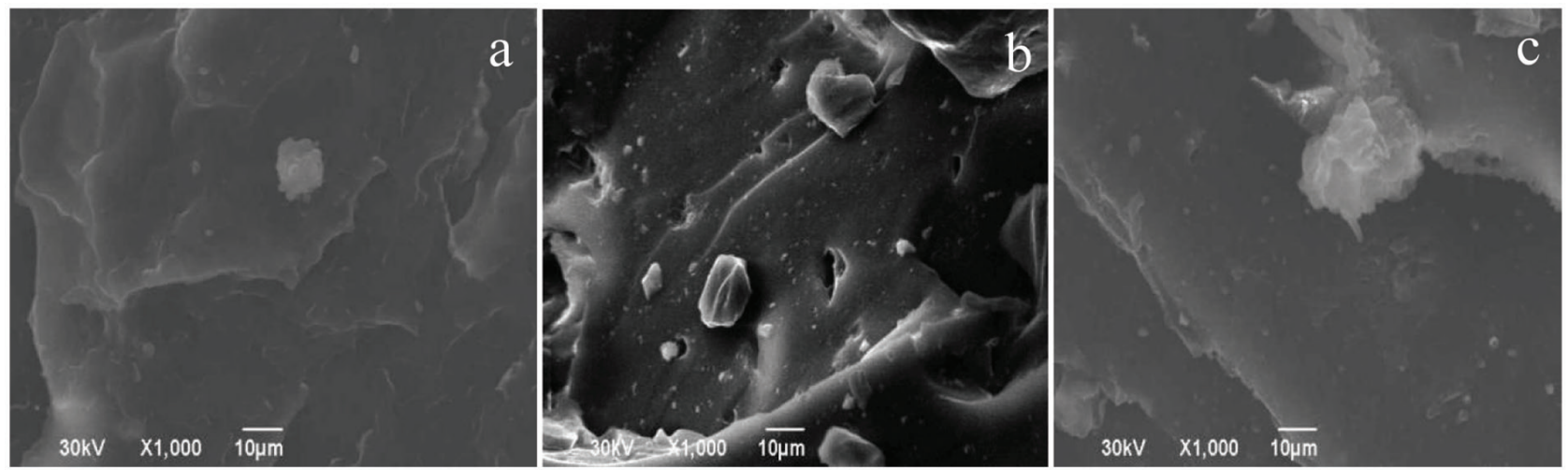

Figure 7: SEM images of RR/NR: (a) 30/70, (b) 50/50, (c) 70/30 blends.

reduced the overall performance of the vulcanizates. Figures $7 \mathrm{a}-\mathrm{c}$ show the fracture surfaces of RR/NR blends, indicating that the surface particles of RR/NR compounds were significantly decreased, and the surface was smoother. This is due to the better compatibility of RR and NR, both RR and NR underwent re-vulcanization molding and formed a continuous phase. It is clear that as the GTR and RR content increased, the GTR/NR compounds exhibits the morphology of brittle fracture, providing evidence for the inferior properties of these compositions. By contrast, the SEM images of the RR/NR blends reveal that the RR homogeneously dispersed in NR matrix, showed considerable deformability, and providing evidence for their superior mechanical properties.

\section{Conclusions}

Curing characteristics showed that compared to the GTR/NR compounds, the scorch time and optimum cure time of $\mathrm{RR} / \mathrm{NR}$ compounds is lower. Compared to the GTR/NR compounds, RR is easy to evenly disperse in NR, and commonly vulcanization to formed a continuous phase, therefore increasing the mechanical properties of RR/NR compounds. DMA measurements showed that with the increase of the RR content, the peak values of the storage and loss modulus of the RR/NR vulcanizates became higher, and the $T g$ value of the RR/NR compounds filled with $90 \mathrm{phr}$ of RR was closer to the high temperature zone than those of the other compositions, thus resulting in the enhancement of the heat resistance properties of the RR/NR composites. SEM studies demonstrated the better interface compatibility of RR/NR compounds, consistent with the mechanical performance test results. Thus, it was showed that thermal-mechanical shearing devulcanization is a useful approach to the use of $R R$ and also provides a feasible route for the incorporation of $R R$ in NR vulcanizates for manufacturing of low-cost products with excellent performance characteristics.

Acknowledgement: The research was supported by the National Natural Science Foundation of China (51273060) and National Undergraduate Training Program for Innovation and Entrepreneurship. 


\section{References}

[1] Shi J., Zou H., Ding L., Li X., Jiang K., Chen T., et al., Continuous production of liquid reclaimed rubber from ground tire rubber and its application as reactive polymeric plasticizer. Polym. Degrad. Stab., 2014, 99, 166-175.

[2] Sienkiewicz M., Kucińska-Lipka J., Janik H., Balas A., Progress in used tyres management in the European Union: a review. Waste. Manage., 2012, 32, 1742-1751.

[3] Sienkiewicz M., Janik H., Borzędowska-Labuda K., KucińskaLipka J., Environmentally friendly polymer-rubber composites obtained from waste tyres: A review. J. Clen. Prod., 2017, 147, 560-571.

[4] Sutanto P., Laksmana F.L., Picchioni F., Janssen L.P.B.M., Modeling on the kinetics of an EPDM devulcanization in an internal batch mixer using an amine as the devulcanizing agent. Chem., Eng., Sci., 2006, 61(19), 6442-6453.

[5] Zhang X., Lu C., Liang M., Properties of natural rubber vulcanizates containing mechanochemically devulcanized ground tire rubber. J. Polym. Res., 2009, 16(4), 411-419.

[6] Ismail H., Omar N., Othman N., Effect of carbon black loading on curing characteristics and mechanical properties of waste tyre dust/carbon black hybrid filler filled natural rubber compounds. J. Appl. Polym. Sci., 2011, 121(2), 1143-1150.

[7] Gao R., Zhang D., Peng S., Preparation of reclaimed rubber by mixing method and secondary vulcanized rubber. China Elastomerics, 2016, 26(3), 12-17.

[8] Zhang D., Xiong J., Peng S., Reinforcement of natural rubber with carbon black/reclaimed rubber hybrid filler. China Elastomerics, 2017, 27(5), 1-6.

[9] Tangudom P., Thongsang S., Sombatsompop N., Cure and mechanical properties and abrasive wear behavior of natural rubber, styrene-butadiene rubber and their blends reinforced with silica hybrid fillers. Mater. Design, 2014, 53(1), 856-864.

[10] Rattanasom N., Saowapark T., Deeprasertkul C., Reinforcement of natural rubber with silica/carbon black hybrid filler. Polym. Test., 2007, 26(3), 369-377.
[11] Thongsang S., Vorakhan W., Wimolmala E., Sombatsompop N., Dynamic mechanical analysis and tribological properties of NR vulcanizates with fly ash/precipitated silica hybrid filler. Tribol. Int., 2012, 53, 134-141.

[12] Jacob M., Francis B., Thomas S., Varughese K.T., Dynamical mechanical analysis of sisal/oil palm hybrid fiber-reinforced natural rubber composites. Polym. Composite., 2006, 27(6), 671-680.

[13] Wu G., Gu J., Zhao X., Preparation and dynamic mechanical properties of polyurethane-modified epoxy composites filled with functionalized fly ash particulates. J. Appl. Polym. Sci., 2007, 105(3), 1118-1126.

[14] Bras J., Hassan M.L., Bruzesse C., Hassan E.A., El-Wakil N.A., Dufresne A., Mechanical, barrier, and biodegradability properties of bagasse cellulose whiskers reinforced natural rubber nanocomposites. Ind. Crop. Prod., 2010, 32(3), 627-633.

[15] Formela K., Haponiuk J.T., Curing characteristics, mechanical properties and morphology of butyl rubber filled with ground tire rubber (GTR). Iran. Polym. J., 2014, 23(3), 1-10.

[16] Jiang H., Kamdem D., Thermal and dynamic mechanical behavior of poly(vinyl chloride)/wood flour composites. J. Appl. Polym. Sci., 2008, 107(2), 951-957.

[17] Pal K., Pal S., Das C., Kim J., Relationship between normal load and dynamic co-efficient of friction on rock-rubber wear mechanism. Mater. Design, 2010, 31(4), 2-9.

[18] Pattanawanidchai S., Saeoui P., Sirisinha C., Influence of precipitated silica on dynamic mechanical properties and resistance to oil and thermal aging in CPE/NR blends. J. Appl. Polym. Sci., 2005, 96(6), 2218-2224.

[19] Thongsang S., Sombatsompop N., Ansarifar A., Effect of fly ash silica and precipitated silica fillers on the viscosity, cure, and viscoelastic properties of natural rubber. Polym. Advan. Technol., 2008, 19(9), 1296-1304.

[20] Theppradit T., Prasassarakich P., Poompradub S., Surface modification of silica particles and its effects on cure and mechanical properties of the natural rubber composites. Mater. Chem. Phys., 2014, 148(3), 940-948. 